being tired with a constant headache which in its and physically co-equal. The child who is carefully incipiency, is generally relieved by rest and out-door taught only such branches as his mind can comprelife. There are many causes in and out of the school hend, and new studies undertaken only when he is room which have a tendency to increase certain dis- competent to understand them will, other things being eases, but our present school system increases nervous equal, accomplish more in the literary world or diseases with all their horrors, often worse than death, achieve greater success in other spheres of life. He and often close in the wake of disordered nervous will be saved untold suffering and not become a bursystems follow tuberculosis which ends the school work of many who promise so much in early life.

The highly sensitive are in great danger from over. work, while those less susceptible do not worry and thus retain their balance, recuperate and throw off influence of school and studies, as soon as at play. How different with the nervous child, who when overtaxed can not throw off anxieties and is constantly expending his nervous energy. We first have slight ailments to treat, but soon the child does not recover from his trouble so easily. The headache becomes more persistent until we finally have congestion of brain, epistaxis, increased digestive trouble with more or less anemia. With the child thus affected his work becomes more irksome and he now requires urging to keep up with the classes. Repeated attacks soon produce a weakness of blood vessels and nerve centers so that slight causes precipitates one of these attacks.

Such children soon become restless and emotional and easily excited or depressed. The girl is now in a condition to be easily afflicted with hysteric trouble which we have so often seen as a climax of school pressure; and the boy thus goaded on does not fare better, for he is in danger of resorting to habits that endangers soul and body.

There can be no doubt that when the sensitive are thus over-worked, we frequently have the epileptic trouble developed. Even if we do not have such serious trouble follow, many are so affected that they are unhappy and unable to compete in life's great battle. Many of our school children are obliged to wear glasses and this is largely due to this same high pressure process.

The eye must have rest, and the child who is compelled to work hard during school hours and until late at night can not thus accommodate it. Thus, not only one organ or function becomes involved by this great strain upon the developing child but every part of the organization is not only endangered but many valuable lives sacrificed in order to satisfy a false delusion called education. With the present tendency to keep adding more branches each year to our course of study we will have more irritable brains to treat with all its sequelæ.

Have any of our really great and strong men or those who have achieved greatness been thus educated? Some of our greatest men only had a knowledge of the common branches, until well matured, and with some practical knowledge of life, a good physical organization and an abundance of good common sense were enabled to honor the highest positions that fame and fortune could willingly and justly bestow.

The present over-pressure process of teaching is having a deleterious effect on our school children in many ways. Not only is disease thus produced directly but we are injuring their reasoning powers in not allowing them to develop into strong physical beings. A child thus overtaxed may arrive at manhood, or womanhood but will never be able to compete with those who are allowed to develop mentally balls was so great they could not be made to recede at 
all, and this pressure was so uniform that voluntary movement of the eye in any direction was impossible. The displacement was directed forward, the eyes looking straight in front. Both anterior chambers were filled with blood, completely obscuring the irides and pupils. Blood was oozing from all portions of the conjunctivæ, but there seemed to be more hemorrhage beneath that portion which lay in the horizontal diameters of the balls, the most profuse being between the external canthi and the corneæ. It was my first impression that there was a double aural hemorrhage, as blood seemed to be flowing from both external auditory canals. But neither the man's position nor condition was favorable to a careful examination at the time.

No evidence of traumatism had been found, except. ing a slight bruise on the forehead where the man had evidently struck the floor, when falling from the lounge. I could find no signs of violence.

The history of the case prior to my arrival was briefly as follows: Mr. M., aged 35 years, who had been in the block some nine to twelve months, was temperate and spent most of his evenings in his rooms reading. For the past three weeks he had been drinking to excess, and for ten days had been intoxicated. On the 8 th he commenced to sober off (taking as he afterward told me 12 ounces of Fellow's hypophosphites in two days). Becoming very nervous and restless on the evening of the 10th, he called in Dr. Clark, who administered $\frac{1}{2}$ grain of codein, which produced a good night's rest. Monday morning, without the knowledge of anyone, he sought the nearest saloon, where he obtained whisky; the exact quantity is not known, but it probably was not very much, for at 12:45, when Dr. Clark called, he was apparently sober.

At 1:10 P.M. he seemed quite well, but nervous; at 1:15 P.M. the people in the vicinity were startled by loud and agonizing screeches. The proprietor of the block at once summoned Dr. Clark, who was in the building. They found Mr. M. on the floor, on his knees and elbows, with a finger in each ear, shrieking with pain. Blood was flowing, apparently, from both eyes and ears, and the eyeballs were protruding. Dr. Clark put him on his back and gave him a hypodermic of morphin, $\frac{1}{4}$ grain. This quieted him in a few min. utes. When I arrived his pulse was 150 , regular, but rather weak; respirations 16. At 2:30 he was given another $\frac{1}{4}$ grain of morphin, his pulse dropped to 120 . He was quiet and his brain, at all times, was sufficiently active to answer all questions intelligently, and he retained perfect use of all his voluntary muscles. The diagnosis was uncertain.

Fearing some exacerbation of pain or further hemorrhage, he was put to bed without being undressed, and given 1 dram doses of fluid extract of ergot every two hours, $\frac{1}{4}$ grain doses of morphin, to keep the pain under control, and 1-100 grain doses of digitalin, hypodermically, to slow and strengthen the heart's action. Locally, hot cloths wrung out of boric acid solution were applied.

At 4 P.M. I again visited the patient. At this time both eyeballs were outside the lids, no motion of balls, but on attempting to move them, there was the faintest movement of the left upper lid. Tension +3 , hemorrhage the same as at first visit, an oozing from the conjunctivæ. An unsatisfactory examination of the ears showed a perfect right tympanum and in the left a mere point in the anterior inferior quadrant, which looked as though it might be a perforation.

On the 12th the eyeballs were beginning to recede and to soften a little. Another examination of the ears was made, which decided that the point was not a perforation but simply a spot between calcareous deposits in the tympanum.

Until this point was decided I had not been able to hazard a diagnosis, but with the decision that the blood which apparently came from the ears was only that which had flowed into them as it trickled down the face from the eyes, I felt that the diagnosis was simplified. At first I thought that there had been an aneurysm of each of the orbital arteries which had ruptured almost simultaneously, due to stimulating effect of the whisky and strychnin.

In the second place, it was possible for an aneurysm of the right orbital artery to exist just after it branches from the internal carotid, within the cranial cavity but extra-dural. This would cause pressure in the right orbital cavity first and later in the left, as the flow of the escaping blood would be in the direction of the least resistance. This theory as to the origin of the trouble at once raised the question: If the hemorrhage was intracranial why did we not have brain symptoms, a slow pulse, impairment of motion to some extent or in some part of the body, disturbed sensation or a clouded intellect? Instead, we found a rapid pulse, 150 at the time of the accident, 120 later in the afternoon, 100 the next day. Motion was perfect and no disturbed sensation. His intellect, while not at its best, was very far from showing indications of a brain lesion. He would answer questions briefly but clearly; his memory, in so far as the accident was concerned up to the $14 \mathrm{th}$, was practically a blank. From an anatomic standpoint, however, I believe it would be possible to have an extra-dural hemorrhage at this point, if the dura mater were sound, which would cause pressure only within the orbital cavities. On the morning of the 14 th the eyes, although causing a hideous expression, had receded considerably, had lost their great tension, and the conjunctivæ and corneæ were breaking down, in fact, pus was present in the anterior chamber of the left eye, but the man's physical condition was growing worse. Fever in the afternoon, pulse rapid and irregular, considerable distress about the orbits but not amounting to actual pain; he was suffering most from his excessively nervous condition.

I advocated the removal of both eyes at once and performed a double enucleation on the 15th. After the operation he began to improve in general vigor, and March 9, about a month from the the date of the accident, he started for his home in Ontario, wearing a pair of "enamels" and feeling as well as he had in years. A couple of days after the operation be gave me the following history: He was a Canadian, single,

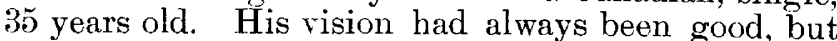
he had suffered periodically from nervous headaches and occasionally had severe pain in left side of head. For six or eight years he had had an external and intermal pterygium of left eye. Occasionally he became intoxicated and on such occasions both eyes would become very much congested and the mucous membrane seemed to extend out over the cheek. but when he would abstain from stimulants his eyes would become normal. One year previous to the accident, while at Seattle, Wash., he had been on a protracted spree, which ended in a condition similar to that for which he sought Dr. Clark's services. He was then 
treated for acute mania and was restored to his normal condition in a couple of weeks. After his attack in Seattle he drank no spirituous liquors for a year, and had on previous occasions abstained four or five years at a time, for he had found by experience that he could not use liquor at all without using too much. During the past year he had been using Warner's Safe Cure, thinking that his kidneys were out of order. For the past three months his health had been poor; everything that he did required an effort and ten days before the accident in order that he might keep up with his work he took some wine. In a few hours he was drunk from the amount of port wine and whisky that he had imbibed, and for seven days remained intoxicated, eating nothing; at the end of that time he drank four bottles of ginger. $\mathrm{He}$ was cold to his knees, his jaws were set and he could not move his arms. After the first drink he seemed to be irresponsible and had no idea of how time passed. After the seven days' spree he stopped drinking absolutely, but was troubled with hallucinations and illusions, against which he struggled for twenty-four hours. Sunday morning he was feeling so weak and

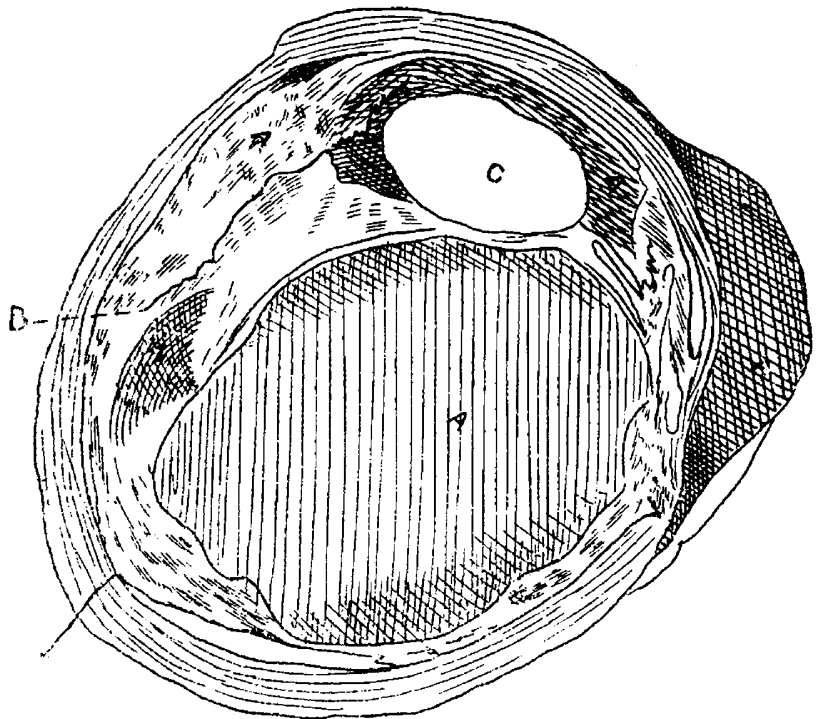

A. hemorrhage: $B$, choroid and retina; C, lens. Retina was also found in hemorrhage.

miserable, with no passage of the bowels in five or six days, he commenced on a bottle of Fellows' hypophosphites and in twenty-four hours had used 12 ounces of it, which represented $13-5$ grains of strychnin. Sunday night he was still feeling so distressed that he called in Dr. Clark, who gave him sufficient codein (I believe) to assure him a good night's rest. Monday he was very excited, and when they brought soup to him at 1 o'clock he would not eat because he thought it was poison. While lying on the lounge he had a spasm of the stomach and with the spasm a blackness came over his eyes; he fell from the lounge, but saw the carpet as he fell. He thought that he had gone to hell and that his brain was full of little devils. With the great pain of the spasm he lost control of himself and he tried to get his fingers into his brain through his eyes, but that caused so much pain that he tried to reach his brain through his ears, then he recollected nothing more till an hour and a half later.

After the eyeballs were removed they were hardened in Wickersham's solution and later in formalin. They were carefully cut, stained and examined micro- scopically by Dr. Alex. Bruce of Edinburgh. Both the eyes were practically the same, and the accompanying diagram is a drawing from one of the sections. This shows the parts very much displaced, the aqueous and vitreous spaces filled with blood, subconjunctival hemorrhage and blood separating choroid and retina from the sclera, with parts of the retina in the hemorrhage. But this gives us no definite information as to the cause of the accident. The pathologist after hearing the imperfect history writes that "the condition was a result of a sudden thrombosis of the sinuses, and of this condition I understand there are some cases on record."

Personally, I am unable to explain the cause of the conditions satisfactorily, although I incline to the theory of his having gouged them out with his thumbs or fingers, but if he did I do not understand why the intra-ocular tension should have been so great. If due to double aneurysm, why was there no aneurysmal bruit and no pulsation, and if due to double thrombosis of the sinuses why should there not be more general disturbance?

\section{REMARKS ON THE CAUSES OF GLAUCOMA.}

Read in the Section on Ophthalmology, at the Forty-seventh Annual Meeting of the American Medical Association, at

BY LEARTUS CONNOR, A.M., M.D. DETROIT, MICH.

Judged by its literature, the causation of glaucoma is unsettled. No effort to harmonize undoubted facts has met with general support. Whether glaucoma is a deformity or a disease, remains an open question. Thus Priestly Smith and his followers claim that glaucoma is a deformity of the eyeball, that an engorgement of the blood vessels in the posterior chamber pushes forward the lens and crowds the ciliary body and iris into the anterior angle, effectually blocking the outlet from the eye for the intraocular secretion. From the resultant intraocular tension he deduces the phenomena of primary glaucoma. The failure of this view to account for glaucomatous attacks in the young, in persons having no iris, in cases of intraocular tumor or dislocated lens, etc., has prevented its universal acceptance.

Of those holding that glaucoma is a disease, part affirm that it is purely local; and part that it is the local expression of a constitutional affection.

Von Graefe called simple glaucoma " amaurosis with excavation," regarding it as quite distinct in origin and course from glaucoma. Iridectomy failed to exert any appreciable influence upon its progress, in contrast with its wonderful power in checking the destructive force of acute inflammatory glaucoma.

Previous to his time glaucoma was regarded as a local inflammation, and treated with antiphlogistios, or as an arthritic disease; the result was the same in either case, viz.: total loss of vision. Not content with the empirical fact that iridectomy would cure a glaucomatous outburst, ophthalmologists have sought out its mechanism and the processes leading to it. The questions raised by this study are most intricate, far-reaching and difficult of solution. Thousands of workers have each contributed something of a positive or negative nature, but much still remains undone. The object of this paper is to briefly discuss a few points bearing upon the causation of glaucoma.

1. An obstruction to the outward flow of the fluids 